\section{Paliperidone Palmitate 6-month (PP6M)}

Paliperidone Palmitate 6-month (PP6M) is an investigational atypical antipsychotic intended for treatment of schizophrenia in a 6-month long-acting injectable (LAI) formulation. Schizophrenia is a chronic brain disorder affecting approximately 20 million people worldwide. It is distinguished by changes in emotions, cognition, perceptions and behaviour and leads to severe disability. Antipsychotic medication plays a prominent role in schizophrenia symptom management. Unfortunately, we are faced with a worldwide problem of nonadherence to prescribed medication, which is frequently the cause of relapse in patients with schizophrenia. LAI antipsychotics enable steady and slow release of the therapeutic agent into the bloodstream, and are therefore superior to oral medications in terms of lack of daily drug administration, and at the same time lower relapse rates and improved patient outcomes [1,2].

Paliperidone palmitate is a LAI formulation of the atypical antipsychotic paliperidone, the primary active metabolite of risperidone. Paliperidone achieves its effect by blockage of dopamine 2 receptors, which in turn reduces positive symptoms of psychosis and also has stabilizing effect on the affective symptoms.
Furthermore, by blocking serotonin $2 \mathrm{~A}$ receptors it enhances dopamine release in certain brain regions and in turn reduces motor side effects and possibly improves cognitive and affective symptoms. Antagonism at the serotonin 7 receptor is mentioned as a possible contributor to the antidepressant actions [3].

The Janssen Pharmaceutical Company announced the submission of PP6M for the treatment of adults diagnosed with schizophrenia to the U.S. Food and Drug Administration in November 2020. The submission is based on the Route 6 Study, a randomized, double-blind, non-inferiority global Phase 3 study of 702 adults with schizophrenia from 20 countries, including Bulgaria, Czech Republic, France, Hungary, Italy, Poland and Spain. It consisted of three phases: a screening phase (up to 28 days), a maintenance phase (of 1 or 3 months), and a double-blind phase (of 12 months). It was designed to demonstrate that injection of PP6M is not less effective than shorter acting formulation of paliperidone palmitate 3-month (PP3M) for the prevention of relapse. Participants were previously stabilized on corresponding doses of PP1M or PP3M (during maintenance phase). Final data revealed non-inferiority of PP6M compared to PP3M on the primary endpoint of time to relapse at the end of the 12-month period in both intent-to-treat and per-protocol analysis sets. The safety profile observed for 


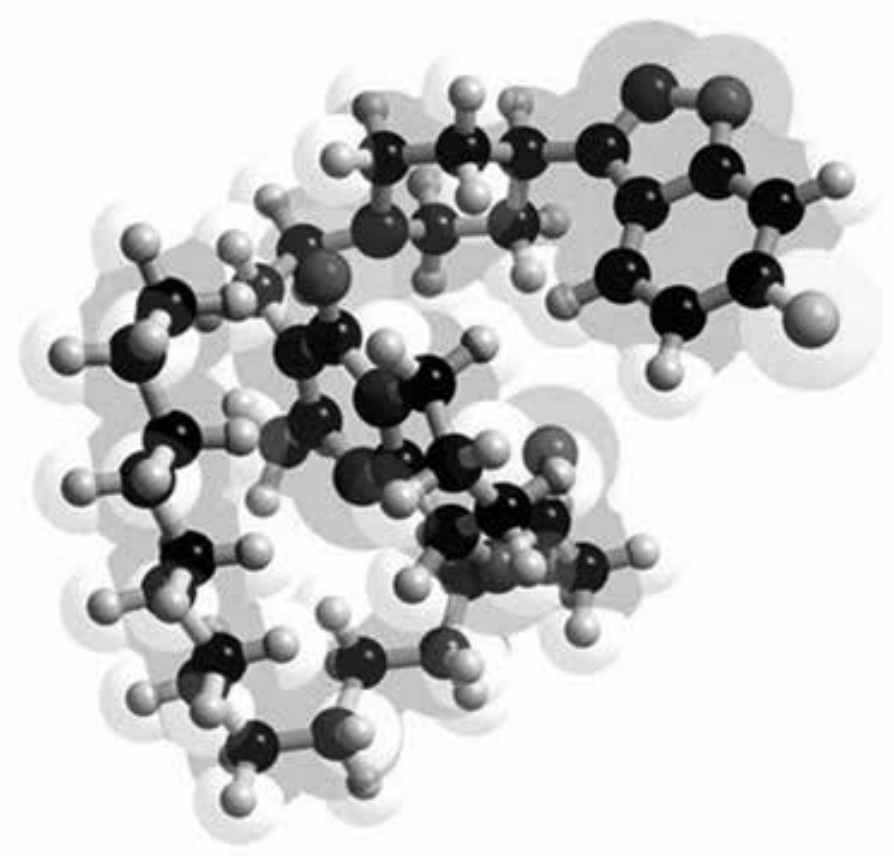

Figure 1. The chemical structure of Paliperidone Palmitate

PP6M was congruous with previous research of paliperidone palmitate shorter acting formulations $[1,4,5]$.

All in all, if approved, PP6M will be the first and only (LAI) schizophrenia medication with a twice-yearly dosing regimen. It is important to emphasise that PP6M is another step towards diminishing nonadherence, which is still a challenging aspect of schizo- phrenia treatment. Needless to say, that kind of a dosing regimen would allow health care workers to devote more resources and time to psychosocial interventions.

Assistant Professor Vjekoslav Peitl, MD, PhD

Darko Vlahovic, $M D$ 


\section{References}

1. Janssen. Janssen Submits Paliperidone Palmitate 6-Month (PP6M) Supplemental New Drug Application to U.S. FDA for Treatment of Schizophrenia in Adults [Internet]. 2021 [cited February 19th]. Available from: https://www.janssen.com/ janssen-submits-paliperidone-palmitate-6-monthpp6m-supplemental-new-drug-application-us-fda

2. Karlović D, Peitl V, Silić A. Shizofrenije. Jastrebarsko: Naklada Slap; 2019.

3. Stahl SM. Stahlovi temelji psihofarmakologije. Jastrebarsko: Naklada Slap; 2017.

4. Businesswire. Janssen Submits Marketing Authorisation Extension to the European Medi- cines Agency to Register Paliperidone Palmitate 6-Monthly (PP6M) for Treatment of Schizophrenia in Adults [Internet]. 2021 [cited February 19th]. Available from: https://www.businesswire.com/news/home/20201204005162/en/ Janssen-Submits-Marketing-Authorisation-Extension-to-the-European-Medicines-Agency-to-Register-Paliperidone-Palmitate-6-Monthly-PP6Mfor-Treatment-of-Schizophrenia-in-Adults

5. Clinicaltrials. A Study of Paliperidone Palmitate 6-Month Formulation [Internet]. 2021 [cited February 19th]. Available from: https://clinicaltrials. gov/ct2/show/NCT03345342 